\title{
Intrapelvic Migration of the Lag Screw with Wedge Wing from Dyna Locking Trochanteric Nail: A Case Report and Literature Review
}

\author{
Yong-Woo Kim, MD*, Weon-Yoo Kim, MD, PhD ${ }^{+, *}$, Kyong-Jun Kim, MD*, Se-Won Lee, MD, PhD*,* \\ Department of Orthopedic Surgery, The Catholic University of Korea, Yeouido St. Mary's Hospital, Seoul, Korea* \\ Department of Orthopedic Surgery, The Catholic University of Korea, Daejeon St. Mary's Hospital, Daejeon, Korea ${ }^{+}$ \\ Department of Orthopedic Surgery, College of Medicine, The Catholic University of Korea, Seoul, Korea ${ }^{\ddagger}$
}

The intrapelvic migration of cervicocephalic lag screws is a rare complication after intertrochanteric fracture synthesis with an intramedullary nail. Only 15 cases of intrapelvic penetration by three different instrument systems have been described in the literature. However, to our knowledge, there is no report of intrapelvic migration of the lag screw with wedge wing designed to increase fixation power using the Dyna locking trochanteric (DLT) nail. We present a case of intrapelvic migration of the lag screw with wedge wing from DLT nail. The patient described herein underwent a two-staged operation of implant removal without intrapelvic approach followed by bipolar hemiarthroplasty. With intrapelvic migration conditions, although it is not uncommon to require an additional intrapelvic approach, this modification can lead to lethal consequences. For this reason, it is recommended to coordinate with the vascular surgery department due to the close proximity of the major vessels.

Key Words: Femur, Intertrochanteric fracture, Complications

Intertrochanteric fracture of the proximal femur is the most common fracture in patients over 65 years of age ${ }^{1)}$; several surgical treatments for this fracture are considered effective at providing early mobilization and low postoperative

Submitted: February 6, 2019 1st revision: April 28, 2019

Final acceptance: May 2, 2019

Address reprint request to

Se-Won Lee, MD, PhD

(https://orcid.org/0000-0002-8467-1957)

Department of Orthopedic Surgery, Yeouido St. Mary's Hospital, College of Medicine, The Catholic University of Korea, 10 63-ro, Yeongdeungpo-gu, Seoul 07345, Korea

TEL: +82-2-3779-1068 FAX: +82-2-783-0252

E-mail: ssewonanaver.com, ssewonagmail.com

This is an Open Access article distributed under the terms of the Creative Commons Attribution Non-Commercial License (http://creativecommons. org/licenses/by-nc/4.0) which permits unrestricted non-commercial use, distribution, and reproduction in any medium, provided the original work is properly cited. morbidity ${ }^{2)}$. Among these treatments, intramedullary fixation devices have recently gained popularity. Various types of intramedullary fixation devices have been developed (e.g., Dyna locking trochanteric [DLT] nail, U\&I, Uijeongbu, Korea; proximal femoral nail antirotation [PFNA], DePuy Synthes, Oberdorf, Switzerland; trochanteric Gamma 3 fixation nail [TFN], Stryker Howmedica, Kalamazoo, MI, USA).

The most common complication associated with use of these implants is cut-out of the hip screw through the femoral head with varus collapse of the fracture ${ }^{3,4}$. Some reports have documented primary medial migration of the lag screw after treatment with intramedullary implants ${ }^{5)}$, but reports of intrapelvic migration of the lag screw are less common. To date, 15 cases of intrapelvic migration have been reported in the literature $\left.{ }^{6-18}\right)$. However, to our knowledge, there are no reports of intrapelvic migration of the lag screw 


\section{Hip \& Pelvis}

Yong-Woo Kim et al. Intrapelvic Migration of the Lag Screw from DLT Nail

with wedge wing designed to increase the fixation power of the DLT nail. We present a case of intrapelvic migration of a lag screw with wedge wing from the DLT nail. We also present a review of the 15 previously reported cases of intrapelvic migration of the lag screw from various intramedullary nail devices in the literature ${ }^{6-18}$, and we review the published outcomes of the DLT nail with wedge wing designed to increase fixation power ${ }^{19-22}$.

Written informed consent was obtained from the patient for the publication of this report and any accompanying images. All of the consent procedure and details were approved by the Institutional Review Board of College of Medicine,
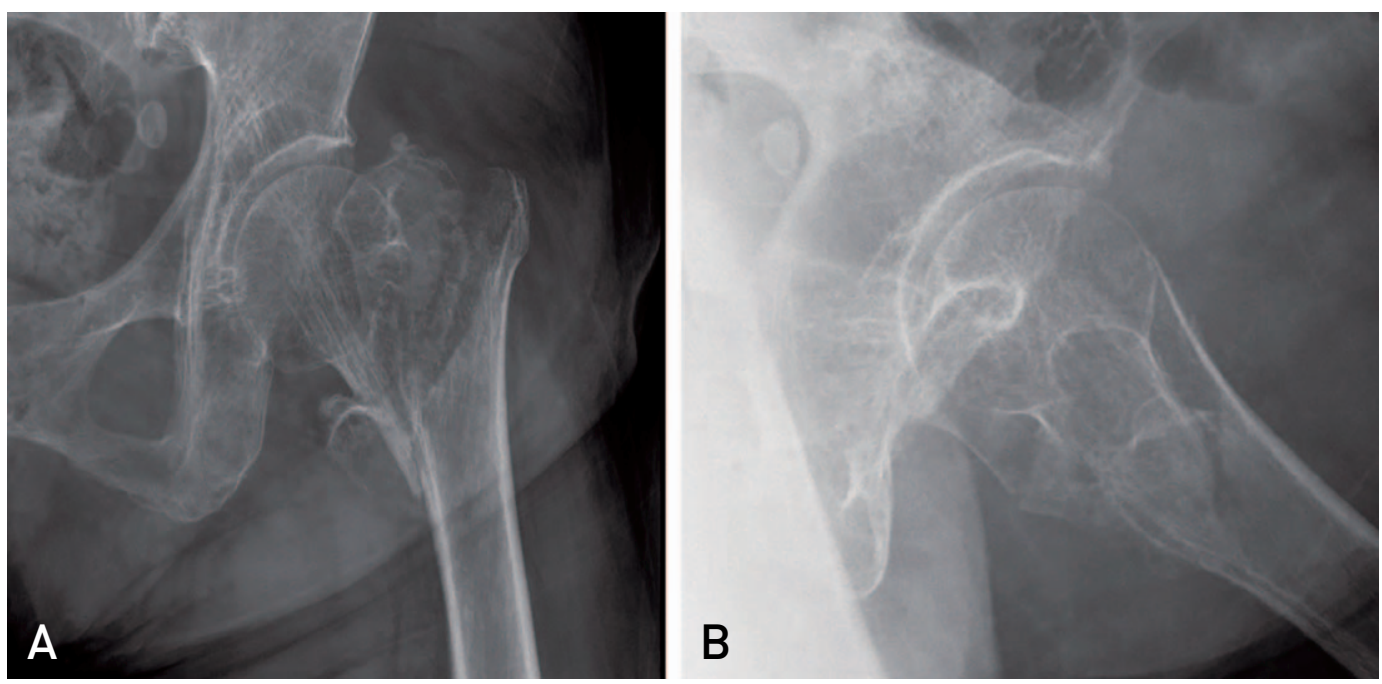

Fig. 1. An 83-year-old woman presented with trauma to the left hip after an accidental fall. On plain radiographs of left femur (A: anteroposterior, B: lateral), an unstable 3-part intertrochanteric fracture was seen (AO/OTA 31-A2).
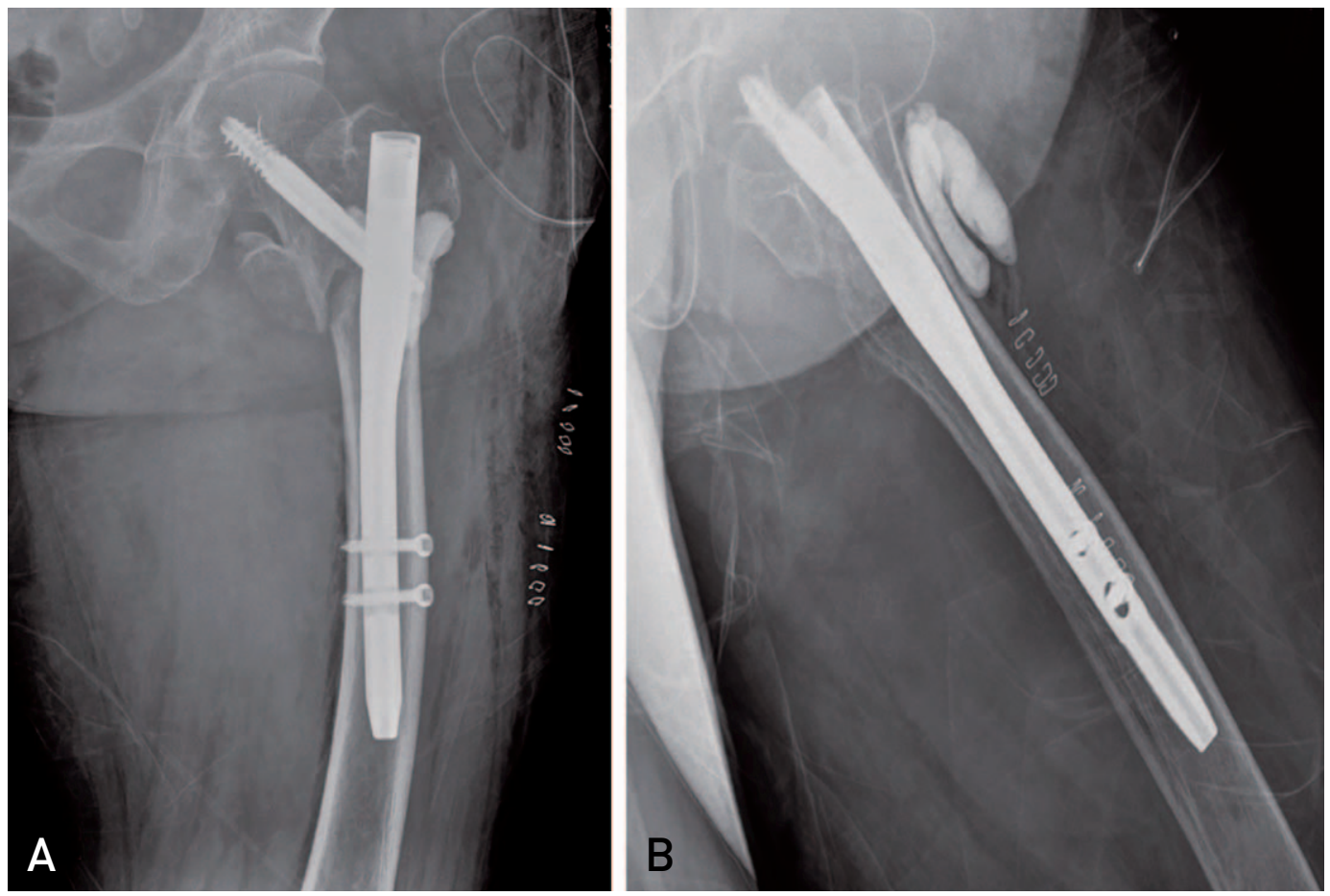

Fig. 2. Postoperative X-ray showing the Dyna locking trochanteric (DLT) nail in the (A) anteroposterior and (B) lateral views. Under general anesthesia on a fracture table, closed reduction was performed, followed by internal fixation with a short (200-mm length) $125^{\circ}$ DLT nail at another local orthopedic clinic. 


\section{Hip \& Pelvis}

Hip Pelvis 31(2): 110-119, 2019

The Catholic University of Korea (DC18ZESI0085).

\section{CASE REPORT}

An 83-year-old woman complained of left hip pain after an accidental fall from standing position onto the ground. On simple radiographs, an unstable intertrochanteric fracture was observed (AO/OTA 31-A2) (Fig. 1). Under general anesthesia, closed reduction was performed on a fracture table, followed by internal fixation with a 200mm length $125^{\circ}$ DLT nail at another local orthopedic clinic (Fig. 2). Walker ambulation was permitted from one week after surgery. The patient was discharged two weeks after the operation. Postoperative radiographs at the two-week follow-up revealed no evidence of implant migration (Fig. 3). Three weeks postoperatively, the patient developed sudden left inguinal pain while ambulating with a walker. She had not experienced any new falls or trauma, and was referred to our clinic. Her radiographs revealed that the lag screw had migrated through the acetabulum, and penetrated into the quadrilateral surface of the pelvis by $4 \mathrm{~cm}$ (Fig. 4A-C). The patient was transferred to our clinic for further management. An abdomen/pelvis computed tomography (CT) with contrast revealed that the lag screw was in close proximity to the external iliac artery and vein (Fig. 4D-F). The radiology report confirmed no evidence of vascular compromise or pseudoaneurysm formation. The patient's vitals remained stable.

We scheduled a two-stage operation. First, the migrated lag screw was removed without injury to other pelvic structures. Immediately after surgery, the pelvis and abdomen were assessed by $\mathrm{CT}$, which revealed no intrapelvic damage due to removal of the migrated lag screw. There was no evidence of intrapelvic compromise, so we decided that it was unnecessary to perform an intrapelvic approach, which would have subjected the patient to an increased risk of postoperative complications, such as infection. However, a vascular surgeon was standing by in the operating room, in the event of an injury to a vessel or abdominal organ during surgery.

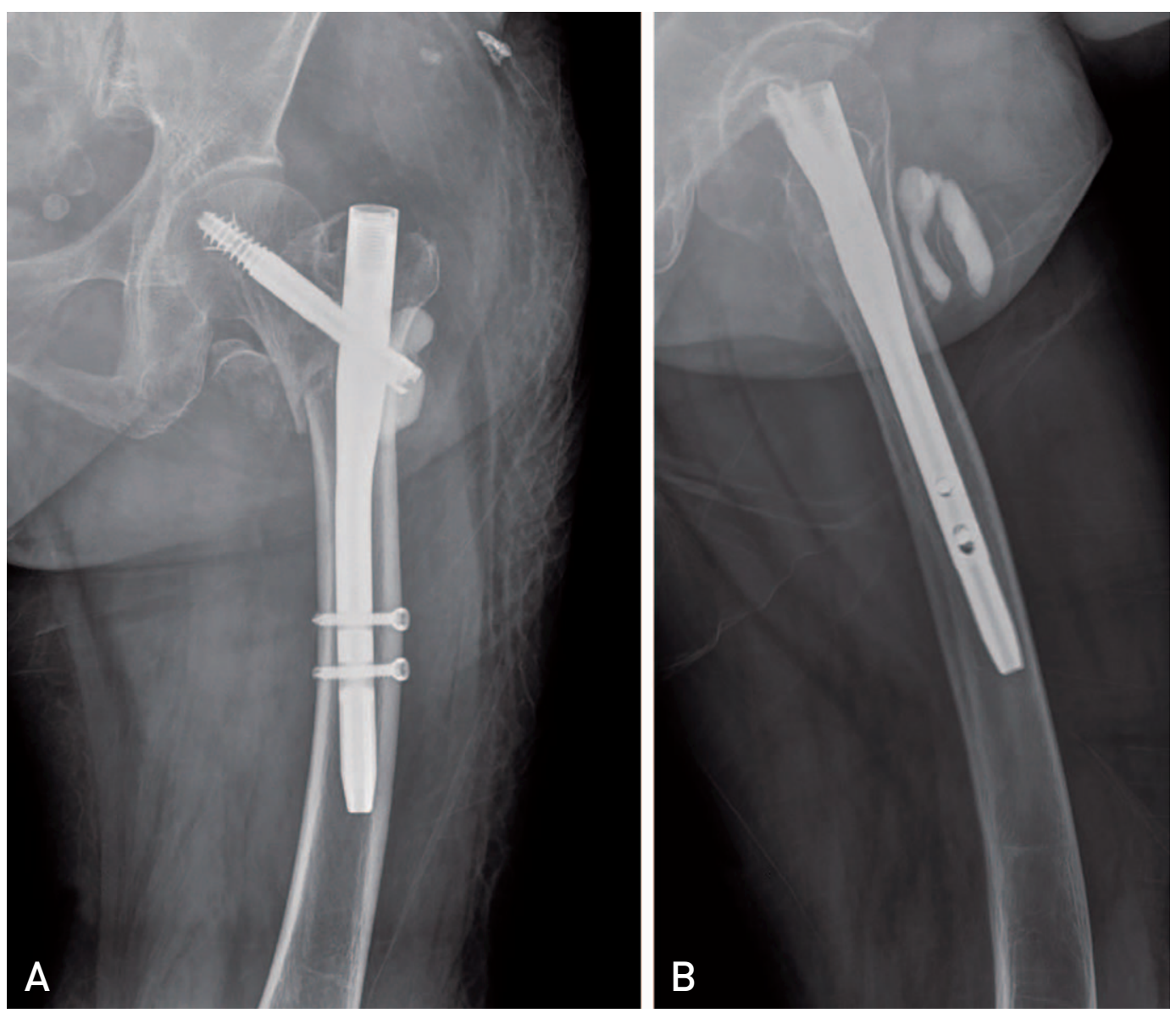

Fig. 3. Postoperative radiographs of left femur (A: anteroposterior, B: lateral) at the 2-week follow-up showed no hardware complications or evidence of migration of the implant. 


\section{Hip \& Pelvis}

Yong-Woo Kim et al. Intrapelvic Migration of the Lag Screw from DLT Nail

Under general anesthesia, the surgery was performed with the patient lying in the lateral decubitus position for the modified Gibson approach. The DLT nail and lag screw were removed under the image intensifier (Fig. 5); in-situ extraction of the femoral head and neck portion was performed. There was no set screw in the nail. There was
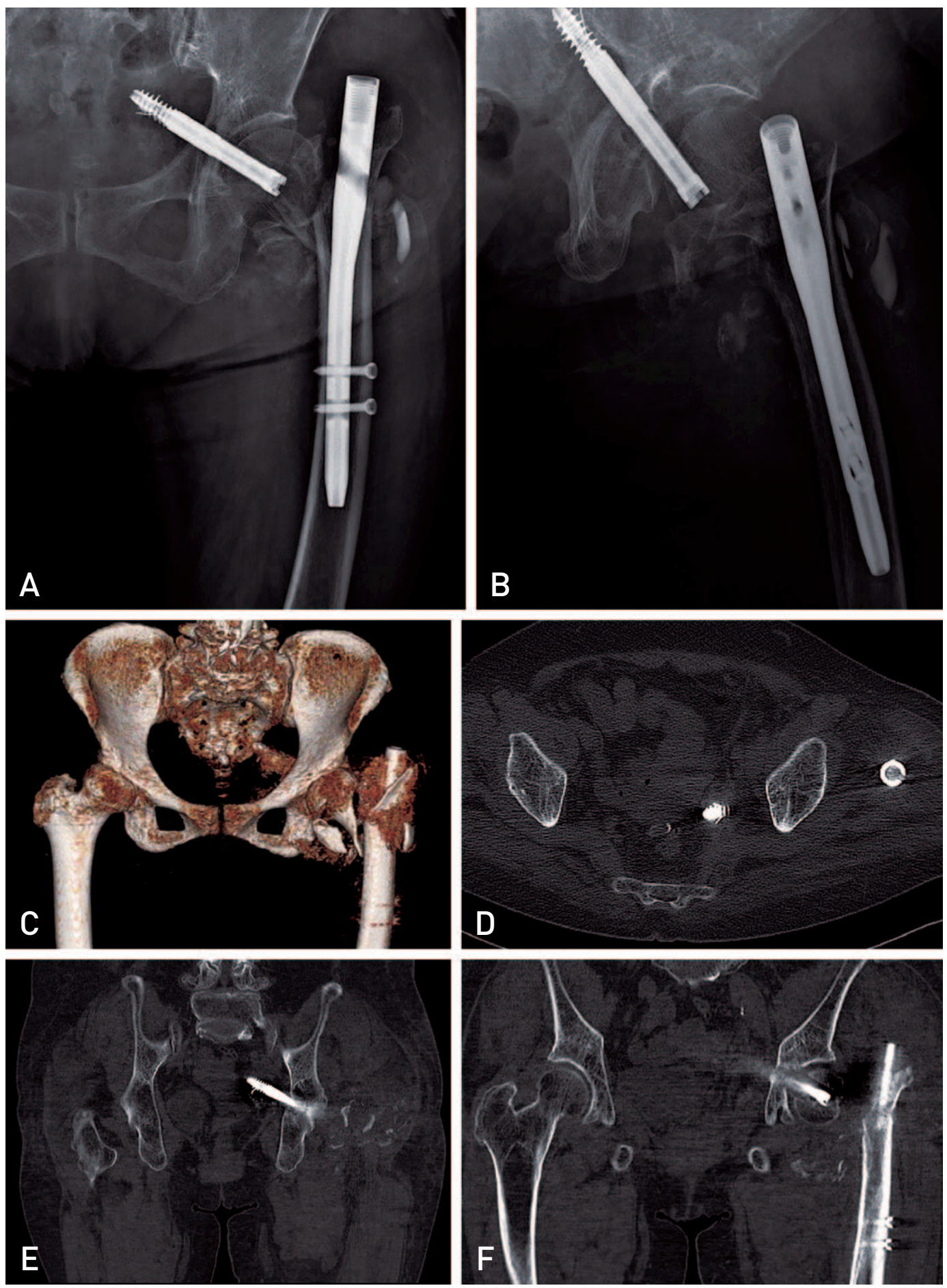

Fig. 4. Radiographs showed that the lag screw was disassociated from the nail and had migrated through the acetabulum. The tip of the lag screw was medial to the quadrilateral surface of the pelvis by about $4 \mathrm{~cm}(\mathrm{~A}, \mathrm{~B})$. Pelvic three-dimensional computed tomography image showed the possibility of an intrapelvic lesion (C), but the hematoma was formed around the lag screw, and the surrounding tissues were not injured (D-F). 


\section{Hip \& Pelvis}

Hip Pelvis 31(2): 110-119, 2019
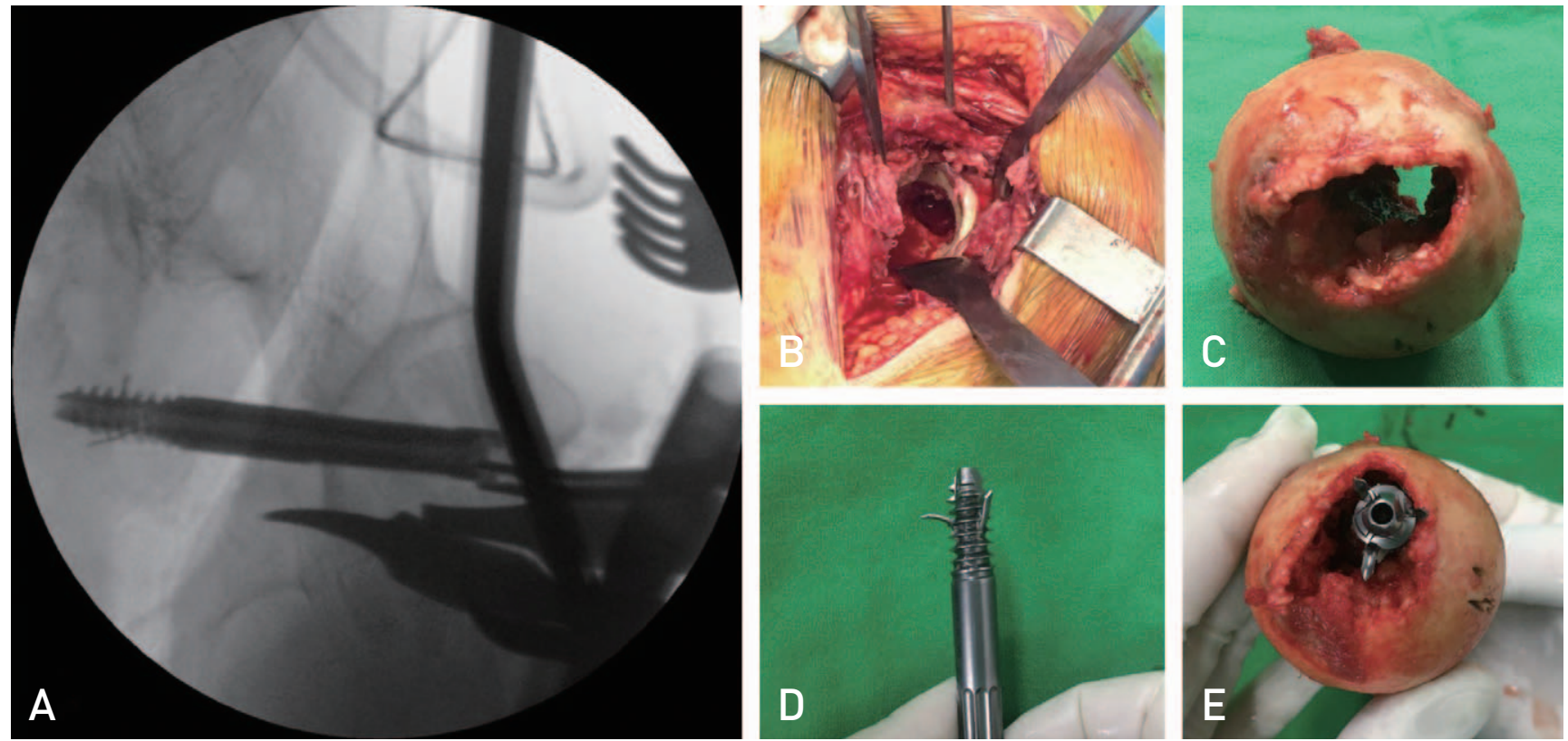

Fig. 5. The penetrated lag screw was removed under image intensifier through the previous incision (A). The penetration defect in the posterior medial acetabulum was observed (B). One of the three wedge wings in the lag screw was folded over and appeared to be located on the inferior side of the extracted femoral head (C-E).
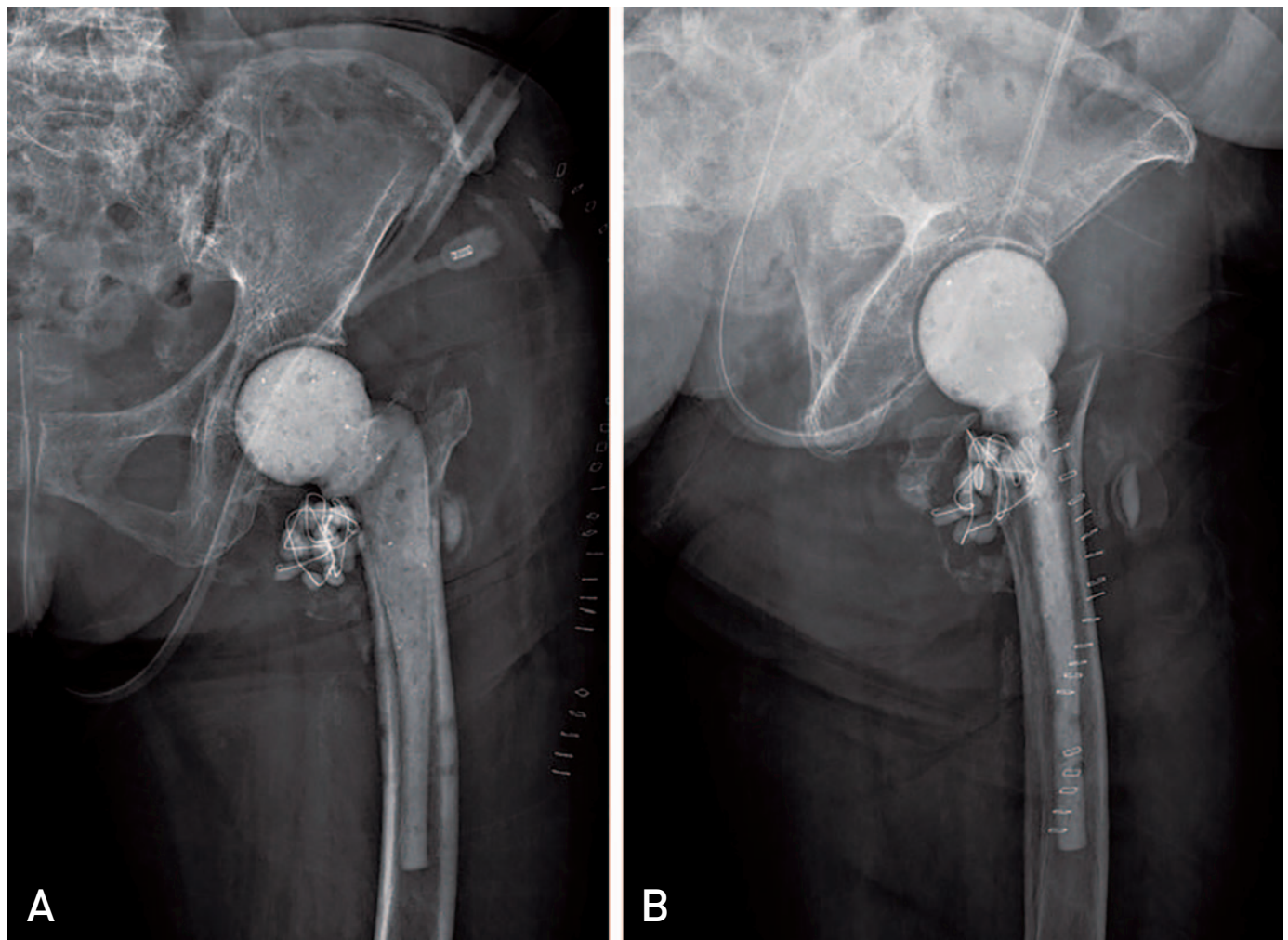

Fig. 6. We scheduled a two-staged operation. After extraction of the femoral head and neck portion, PROSTALC (prosthesis with antibiotic-loaded acrylic cement) was performed at the first scheduled surgery to maintain leg length and reduce the risk of infection. Postoperative radiographs of left femur (A: anteroposterior, B: lateral) 


\section{Hip \& Pelvis}

Yong-Woo Kim et al. Intrapelvic Migration of the Lag Screw from DLT Nail

a penetrated defect in the posterior medial acetabulum. One of the three wedge wings in the lag screw was folded over and appeared to have been located on the inferior side of the extracted femoral head (Fig. 5).

To maintain leg length and reduce the risk of infection, PROSTALC (prosthesis with antibiotic-loaded acrylic cement) was performed (Fig. 6). After confirming that cultures and tissue samples were negative for infection, we planned the second operation. According to a previous study, the best salvage procedure for cut-out after surgical fixation of trochanteric fractures with the PFNA or TFN is total hip arthroplasty ${ }^{23)}$; however, based on clinical experience, we performed the hemiarthroplasty as the patient had severe dementia and relative insufficiency of the abductor musculature, the penetrated area was not in the weight bearing portion, and cut-through progressed toward acute.

In the second operation conducted four weeks after the removal surgery, an uncemented bipolar hip arthroplasty (Modulus; Limacorporate S.p.A., Udine, Italy) and application of a long $\mathrm{AO}$ trochanteric reattachment device for the fractured greater trochanteric fragment were performed (Fig. 7). The patient was allowed full weight bearing immediately postoperatively and she was discharged to a rehabilitation facility two weeks postoperatively. The latest clinical and radiologic follow-up at 10 months revealed uneventful healing, and the patient was able to walk without walking aids.

\section{DISCUSSION}

Intertrochanteric fracture of the proximal femur is the most common fracture in patients over 65 years of age. Intramedullary fixation devices have gained popularity, but they are associated with some potential complications, the most common of which is cut-out of the hip screw through the femoral head with varus collapse of the fracture $^{3,4)}$. However, there is a paucity of literature about intrapelvic migration of the lag screw.

Werner-Tutschku et al. ${ }^{24)}$ were the first to report medial migration of the lag screw from a proximal femoral nail (PFN; DePuy Synthes). They demonstrated the "Z-effect" as a cause of medial migration of the proximal lag screw, combined with lateral migration of the lag screw, in $7.1 \%$ of their patients. They reported that the most important factor for migration was fracture varus malreduction prior
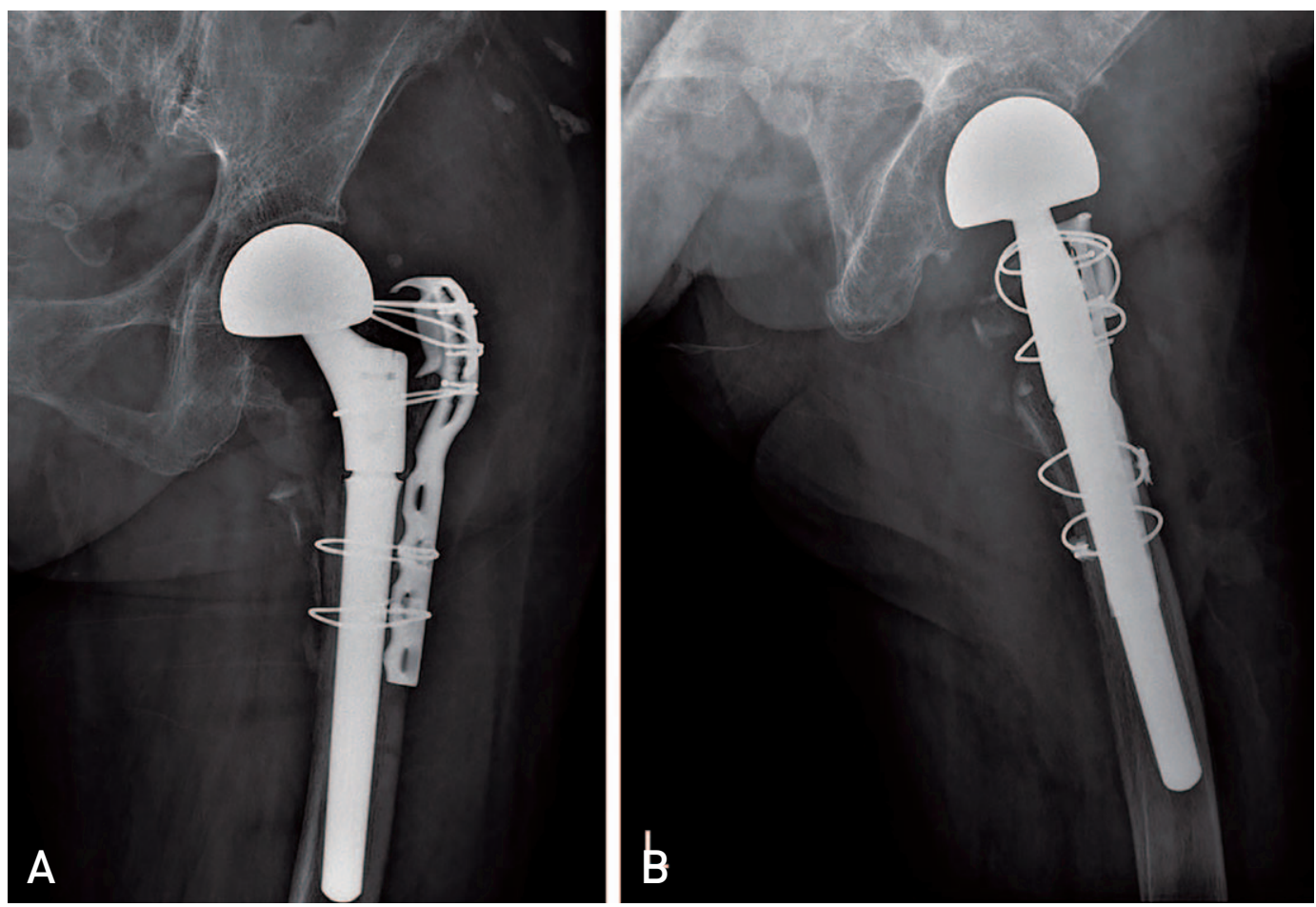

Fig. 7. Postoperative radiographs of left femur (A: anteroposterior, B: lateral). In the second operation at 6 weeks after the removal surgery, an uncemented bipolar hip arthroplasty (Modulus; Limacorporate S.p.A., Udine, Italy) and application of a long AO trochanteric reattachment device (DePuy Synthes, Oberdorf, Switzerland) for the fractured greater trochanteric fragment were performed. 


\section{Hip \& Pelvis}

Hip Pelvis 31(2): 110-119, 2019

to nail insertion. Two cut-outs and five Z-effects were observed in 12 patients with a caput-collum-diaphyseal (CCD) angle less than $125^{\circ}$ after fracture reduction process, compared to only one cutout and no Z-effect in 58 patients with a CCD angle more than $125^{\circ}$. This type of migration has been described in for two screw fixation devices (i.e., PFN). In single lag screw fixation devices, a few cases of medial intrapelvic migration of the lag screw have been reported.

Several factors can contribute to medial migration of a lag screw. In a study of Rebuzzi et al. ${ }^{25}$ that included 981 intramedullary hip fracture fixations using Intramedullary Hip Screws (IMHS; Smith \& Nephew Richards, Memphis, TN, USA), a 'risky' screw position was evident in 21 of these cases, along with nine lag screws cut out, with three penetrating the acetabulum. These data reveal that medial migration is a rare $(<1 \%, 9 / 981)$ complication of the total number of intramedullary hip fracture fixations, but it is relatively common when the screw position is deemed to be risky $(42.9 \%, 9 / 21)$. Thein et al. ${ }^{\circ}$ categorized risk factors of lag screw medial migration into risk related to fracture pattern and risk related to operative technique.

Until now, 15 cases of intrapelvic migration have been reported in the literature ${ }^{6-18}$ (Table 1). Intrapelvic migration of a lag screw occurred between 19 days and 11 months postoperatively, and five of the 15 cases occurred within three months postoperatively. All cases except one revealed no definite trauma history. All cases were in patients aged 63 years or older (mean, 74.7 years; median, 75 years), except for one (40 years old). Most (12 of 15) of the reported cases of intrapelvic migration of a lag screw involved gamma nail use $^{6-8,11-15,17,18)}$. The other three cases involved a trochanteric nail $^{10)}$ (DePuy ACE, Warsaw, IN, USA), PFN (DePuy Synthes) ${ }^{16)}$, or IMHS ${ }^{9}$. Most authors (11 of 15) chose to convert to arthroplasty. In three cases ${ }^{13,14,16)}$, the implant was removed, and in one of these cases, the patient died of lethal hemorrhage during implant removal ${ }^{15}$. In one case, a migrated lag screw was changed to a shorter one, and an additional screw was inserted to obtain fusion ${ }^{14)}$.

When we encounter intrapelvic migration, we are very

Table 1. Pooled Data of Published Case Reports* on Intrapelvic Migration of a Lag Screw from a Cephalomedullary Nail

\begin{tabular}{|c|c|}
\hline Variable & Data \\
\hline \multicolumn{2}{|l|}{ Age (yr) } \\
\hline Mean & 74.7 \\
\hline Median (range) & $75(40-92)$ \\
\hline Sex, male/female & $6 / 9$ \\
\hline Fracture type, $\mathrm{A} 1 / \mathrm{A} 2 / \mathrm{A}^{+}$ & $1 / 9 / 5$ \\
\hline \multicolumn{2}{|l|}{ Implant } \\
\hline Gamma nail & $12^{\neq}$ \\
\hline PFN & 1 \\
\hline Trochanteric nail & 1 \\
\hline IMHS & 1 \\
\hline Time to failure & $2 \mathrm{mo}(19 \mathrm{~d}-11 \mathrm{mo})$ \\
\hline$\leq 3 \mathrm{mo}$ & 10 \\
\hline$>3 \mathrm{mo}$ & 5 \\
\hline \multicolumn{2}{|l|}{ Trauma history } \\
\hline Definite trauma & 1 \\
\hline No trauma & 14 \\
\hline \multirow[t]{2}{*}{ Additional intrapelvic approach } & Intrapelvic approach, 2 \\
\hline & Embolization of a branch of the internal iliac artery, 1 \\
\hline \multirow[t]{3}{*}{ Additional surgery } & Converted to arthroplasty, $11^{\text {s }}$ \\
\hline & Implant removed, 3 (including 1 case of death) \\
\hline & Change of lag screw and insertion of additional screw, 1 \\
\hline
\end{tabular}

Values are presented as number only or median (range).

* Total of 15 cases from 13 reports.

+ AO classification.

${ }^{*}$ Gamma third generation: 8, previous generations: 4 .

${ }^{\S}$ One-staged operation: 10, two-staged operation: 1.

PFN: proximal femoral nail (DePuy Synthes, Oberdorf, Switzerland); Trochanteric nail: DePuy ACE, Warsaw, IN, USA; IMHS: intramedullary hip screws (Smith \& Nephew Richards, Memphis, TN, USA). 


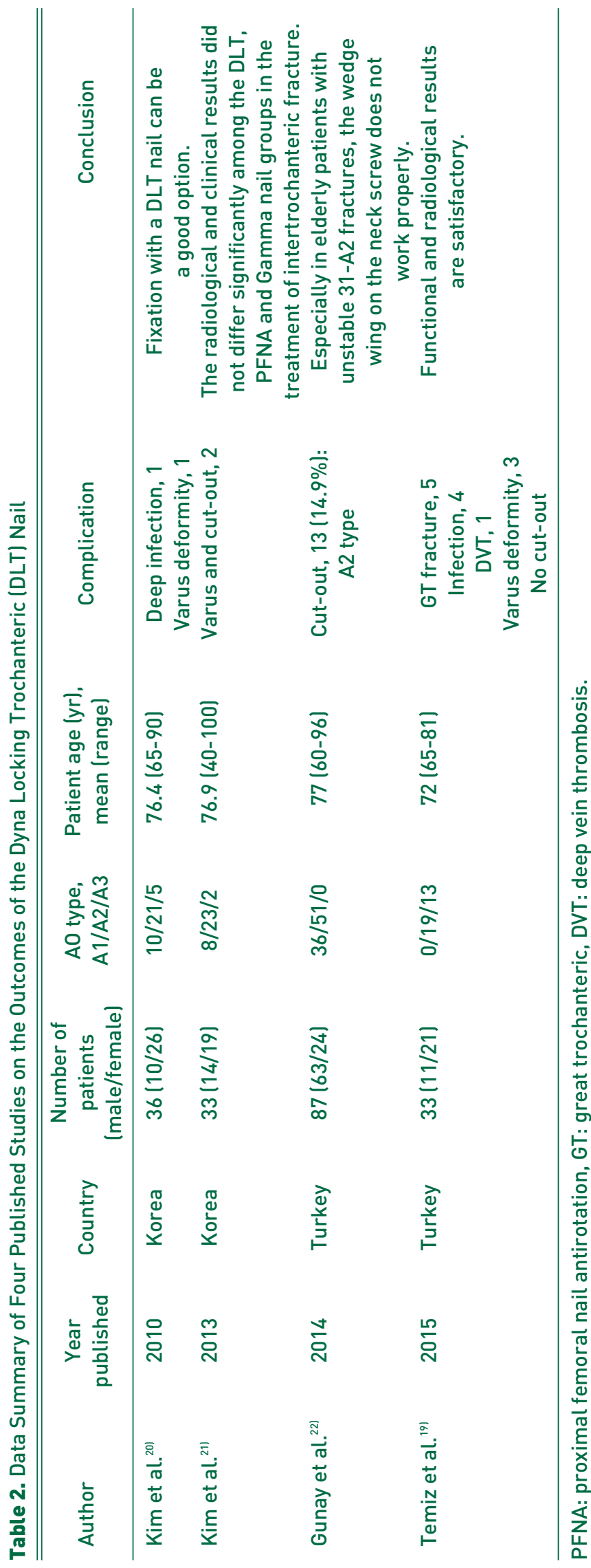

careful to assess whether the intrapelvic organ is damaged. It is necessary to prepare for a risky intraoperative situation through the cooperation of other departments. Fortunately, in our case, a hematoma formed around the lag screw, and the surrounding tissues were not injured. In the literature, two cases were treated with an intrapelvic approach, but another case underwent embolization of a branch of the internal iliac artery ${ }^{6,9,10)}$. Tauber and Resch $^{7}$ reported on late-recognized sigmoid perforation caused by intrapelvic migration of the lag screw. Their patient developed retroperitoneal abscess formation 12 weeks after conversion to total hip arthroplasty due to undetected sigmoid perforation from the migrated lag screw, and additional abdominal surgery was needed. Recently, Lee et al. ${ }^{15}$ reported a case of intrapelvic migration that led to death. In their case, the proximal portion of the lag screw was located in the retroperitoneal area. Massive bleeding due to vessel injury during screw removal was presumed to have led to death. Surgeons need to be well prepared before surgery for the worst possible situation.

The DLT nail is a cannulated implant, consisting of titanium alloy with a proximal curvature of $6^{\circ}$. The proximal part of the implant is $16.5 \mathrm{~mm}$ in diameter and has a lag screw with a diameter of $11 \mathrm{~mm}$. The DLT nail has three wedges at the tip of the lag screw. After the lag screw is inserted, the wedge wing in the lag screw is opened to prevent twisting and rotation of the screw in the femoral head. The wedge wings have been designed to hold the femoral head more rigidly and improve axial stability.

Four reports have described the use of the DLT nail in Turkey and Korea ${ }^{19-22)}$ (Table 2). Pooling the data from these four reports, there were a total of 188 patients. Among these,

Table 3. Pooled Data from Published Studies* on the Outcomes of the Dyna Locking Trochanteric Nail

\begin{tabular}{lc}
\hline \hline Variable & Data \\
\hline Number of studies & 4 \\
Number of patients & 188 \\
Mean age (yr) & 76 \\
Sex, male/female & $59 / 129$ \\
A0 type, A1/A2/A3 & $54 / 114 / 20$ \\
Complication & 34 (18.1) \\
Varus and cut out & 19 \\
Infection & 5 \\
Deep vein thrombosis & 1 \\
Great trochanteric fracture & 5 \\
Heterotopic ossification & 4 \\
\hline * Total of 188 patients from four studies. &
\end{tabular}


there were 34 complications (18.1\%), including cut-out (19 patients), infection (5 patients), deep vein thrombosis (1 patient), intraoperative greater trochanteric fracture (5 patients), heterotopic ossification (4 patients) (Table 3 ). Gunay et al. ${ }^{22)}$ observed cut-out complications in 13 of 51 (25.5\%) elderly patients with unstable fractures (31-A2) treated with a DLT nail. Although the authors found that cut-out was closely related to the position of the lag screw, reduction quality, and an unstable fracture pattern, they concluded that the wedge wings in the lag screw do not work properly in patients with poor bone quality. In our case, during the removal procedure, it was observed that no set screw was inserted to fix the lag screw to the nail shaft during the initial operation. In addition, one of the three wedge wings was bent. We assumed that it was bent by a locally hardened area of the femoral head during the process of opening the wedge wings, resulting in medial migration due to a phenomenon similar to the $\mathrm{Z}$ effect ${ }^{233}$. In addition, initial reduction quality was not good, which showed inappropriate alignment anterior cortex, and the operation was not performed according to the manufacturer's guidelines whose process of inserting the set screw was omitted, which may have contributed to the occurrence of this complication.

When we encounter intrapelvic migration conditions, we are very careful to assess whether the intrapelvic organ has been damaged. Before surgery, it is necessary to check for vascular damage by checking the intervention image, and the location of the vessels can be checked to predict the possibility of major vascular damage during surgery. In addition, it is thought that the surgery after asking for cooperation from the vascular and imaging departments prior to surgery will help repair the damage quickly in the event of vascular damage during surgery.

\section{ACKNOWLEDGEMENTS}

We thank the patient for providing consent for this case report.

\section{CONFLICT OF INTEREST}

The authors declare that there is no potential conflict of interest relevant to this article.

\section{REFERENCES}

1. Kenzora JE, McCarthy RE, Lowell JD, Sledge CB. Hip fracture mortality. Relation to age, treatment, preoperative illness, time of surgery, and complications. Clin Orthop Relat Res. 1984;(186):45-56.

2.Hardy DC, Descamps PY, Krallis P, et al. Use of an intramedullary hip-screw compared with a compression hip-screw with a plate for intertrochanteric femoral fractures. A prospective, randomized study of one hundred patients. J Bone Joint Surg Am. 1998;80:618-30.

3. Chevalley F, Gamba D. Gamma nailing of pertrochanteric and subtrochanteric fractures: clinical results of a series of 63 consecutive cases. J Orthop Trauma. 1997;11:412-5.

4. Hesse B, Gächter A. Complications following the treatment of trochanteric fractures with the gamma nail. Arch Orthop Trauma Surg. 2004;124:692-8.

5. Weil YA, Gardner MJ, Mikhail G, Pierson G, Helfet DL, Lorich DG. Medial migration of intramedullary hip fixation devices: a biomechanical analysis. Arch Orthop Trauma Surg. 2008; 128:227-34.

6. Thein E, De Cannière A, Burn A, Borens O. Medial migration of lag screw after gamma nailing. Injury. 2014;45:1275-9.

7. Tauber M, Resch H. Sigmoid perforation after medial migration of lag screw in gamma nailing. Arch Orthop Trauma Surg. 2006; 126:118-22.

8. Takasago T, Goto T, Toki S, et al. Intrapelvic migration of the lag screw in intramedullary nailing. Case Rep Orthop. 2014;2014:519045.

9. Robinson SJ, Fountain JR, Torella F, Pennie BH. Intrapelvic migration of a lag screw from a cephalomedullary femoral nail: a case report. Injury. 2011;42:1384-6.

10. Ramkumar U, Saffar N, Thinakarajan T, Parmar HV. Pelvic migration of lag screw from a nailing device. Inj Extra. 2006;37:53-5.

11. Pinheiro AC, Alpoim B, Félix A, Alves C, Sousa C, Rodrigues A. Medial migration of the intramedullary Gamma 3 nail a case report. Rev Bras Ortop. 2016;51:720-4.

12. Lucke M, Burghardt RD, Siebenlist S, Ganslmeier A, Stöckle U. Medial migration of lag screw with intrapelvic dislocation in gamma nailing--a unique problem? A report of 2 cases. J Orthop Trauma. 2010;24:e6-11.

13.Lozano-Alvarez C, Alier A, Pelfort X, Martínez-Díaz S, Puig L. Cervicocephalic medial screw migration after intertrochanteric fracture fixation, OTA/AO 31-A2, using intramedullary nail Gamma3: report of 2 cases and literature review. J Orthop Trauma. 2013;27:e264-7.

14. Li X, Heffernan MJ, Kane C, Leclair W. Medial pelvic migration of the lag screw in a short gamma nail after hip fracture fixation: a case report and review of the literature. J Orthop Surg Res. 2010;5:62.

15. Lee JW, Cho HM, Seo JW. Intrapelvic penetration of lag screw in proximal femoral nailing: a case report. J Korean Fract Soc. 2017;30:203-8.

16. Lal H, Sharma DK, Mittal D. Intrapelvic migration of hip lag screw of proximal femoral nail-sequele to a paradoxical reverse $Z$ effect and their critical analysis. J Clin Orthop Trauma. 2012;3:48-53.

17. Heineman DJ, van Buijtenen JM, Heuff G, Derksen EJ, Pöll RG. Intra-abdominal migration of a lag screw in gamma nailing: report of a case. J Orthop Trauma. 2010;24:e119-22.

18. Flint JH, Sanchez-Navarro CF, Buckwalter JA, Marsh JL. 


\section{Hip\& Pelvis}

Yong-Woo Kim et al. Intrapelvic Migration of the Lag Screw from DLT Nail

Intrapelvic migration of a gamma nail lag screw: review of the possible mechanisms. Orthopedics. Published online April 1, 2010; doi: 10.3928/01477447-20100225-19.

19. Temiz A, Durak A, Atici T. Unstable intertrochanteric femur fractures in geriatric patients treated with the DLT trochanteric nail. Injury. 2015;46 Suppl 2:S41-6.

20. Kim YS, Yoon JW, Han SK. Treatment of intertrochanteric fracture of the femur using a dyna locking trochanteric (DLT) nail. J Korean Hip Soc. 2010;22:216-21.

21. Kim SS, Lee KY, Kim CH, et al. Comparison of the dyna locking trochanteric nail, proximal femoral nail antirotation and gamma 3 nail in treatment of intertrochanteric fracture of the femur. Hip Pelvis. 2013;25:211-9.

22. Gunay C, Atalar H, Altay M, Yavuz OY, Yildirim AO, Oken OF. Does the wedge wing in the neck screw prevent cut-out failure in Ota/AO 31-A2 trochanteric fractures in elderly patients? Acta Orthop Belg. 2014;80:26-33.

23. Brunner A, Büttler M, Lehmann U, et al. What is the optimal salvage procedure for cut-out after surgical fixation of trochanteric fractures with the PFNA or TFN?: a multicentre study. Injury. 2016;47:432-8.

24. Werner-Tutschku W, Lajtai G, Schmiedhuber G, Lang T, Pirkl C, Orthner E. [Intra- and perioperative complications in the stabilization of per- and subtrochanteric femoral fractures by means of PFN]. Unfallchirurg. 2002;105:8815. German.

25. Rebuzzi E, Pannone A, Schiavetti S, et al. IMHS clinical experience in the treatment of peritrochanteric fractures. The results of a multicentric Italian study of 981 cases. Injury. 2002;33:407-12. 